\title{
POPULAÇÕES DE MILHO: CARACTERÍSTICAS AGRONÔMICAS E TECNOLÓGICAS (')
}

\author{
MARLENE LIMA $(2,5)$, POLICARPO VITTI $\left({ }^{3,5}\right)$ e PAULO BOLLER GALLO $(4,5)$
}

\section{RESUMO}

Avaliaram-se duas populaçōes de milho de grãos brancos (IACTaitinga e IAC-Moroti) e duas de grãos amarelos (IAC-Taiúba e IAC-lubatã) e os cruzamentos recíprocos entre as de grãos brancos e três testemunhas (Ag-401, C-601 e IAC-Porangatu), em dois locais, quanto a características agronômicas. Com exceção das testemunhas, testaram-se também tais populaçōes quanto aos principais parâmetros de interesse tecnológico, relacionados com o rendimento e qualidade do fubá mimoso, para uso em panificação. Foram observadas diferenças altamente significativas entre os tratamentos para os caracteres produitividade de grăos, altura da planta e de inserção da espiga, rendimento de gérmen e de fubá mimoso, volume especifico comparativo (V.E.C.) e escore total comparativo (Es. T.C.). As populações, de modo geral, apresentaram altos niveis de produção de grãos, sendo o maior valor obtido pela população IAC-Taitinga. Sete dos materiais estudados apresentaram porte médio e dois, porte baixo. A população IAC-

(1) Parciaimente financiado pela Fundação Cargill. Apresentado no Xvi Congresso Nacional de Milho e Sorgo, Belo Horizonte, MG, 4 a 8 de agosto de 1986. Recebido para publicação em 29 de outubro de 1986 e aceito em 16 de março de 1988. nas (SP).

(2) Seção de Genética, Instituto Agronômico (IAC), Caixa Postal 28, 13001 Campinas (SP).

(3) Seção de Panificaçāo, Instituto de Tecnologia de Alimentos (ITAL), Caixa Postal 139, 13001 Campi-

$\left({ }^{4}\right)$ Estação Experimental de Mococa, IAC.

(5) Com bolsa de pesquisa do CNPq. 
Taitinga, sem diferir de IAC-Moroti e do híbrido IAC-Taitinga x IAC Moroti, apresentou o maior valor para rendimento de fubá mimoso. A IAC-Taiúba sobressaiu-se das demais quanto ao V.E.C. e só não alcançou valor máximo para Es.T.C. devido à cor amarela dos grãos. A IAC-Taitinga e o híbrido IAC-Taitinga x IAC-Moroti mostraram valores iguais ao padrão (100\%) para Es.T.C. Foi observado efeito maternal atuando no rendimento do fubá mimoso obtido.

Termos de indexação: Zea mays L.; melhoramento genético; efeito maternal; panificação; farinha mista.

\section{INTRODUÇĀO}

Entre os cereais, o milho e o trigo são considerados dos mais importantes, sendo o primeiro utilizado, principalmente, na alimentação animal e o segundo, na alimentação humana. O milho é largamente cultivado no Brasil porque se adapta a uma ampla variedade de condições edafoclimáticas, enquanto as áreas próprias ao cultivo do trigo são mais restritas. Este fato situa o milho como o cereal de maior produção de grãos (em tomo de vinte milhões de toneladas anuais), tornando o País auto-suficiente em anos normais. Entretanto, no caso do trigo, sempre houve necessidade de importaçōes anuais de grandes quantidades para complementar a produção nacional.

Com o objetivo de reduzir a dependência externa de trigo, pesquisas desenvolvidas no Instituto de Tecnologia de Alimentos (ITAL) (VITTI et al., 1980) demonstraram que é possivel, no processo de panificação, a adição de farinha de milho integral e desengordurada à farinha de trigo, na proporção de $25 \%$, sem alteraçóes aparentes na qualidade do produto final.

Os programas de melhoramento genético de milho já produziram híbridos e cultivares de excelente potencial produtivo e com caracteres agronômicos adequados a nossas condições ambientais. Todavia, pouca ou nenhuma ênfase tem sido dada ao estudo de parâmetros de qualidade do produto, principalmente aqueles relacionados com a fabricação de massas e pães.

O objetivo do presente trabalho foi avaliar populações e híbridos de mitho quanto a algumas características de interesse agronômico, rendimento de fubá mimoso, rendimento de gérmen e avaliaçāo física dos pães preparados.

\section{MATERIAL E MÉTODOS}

A população IAC-Taiúba, obtida pelo intercruzamento de treze subpopulaçōes, doze híbridos e uma variedade, apresenta plantas de porte médio, ciclo intermediário e bom potencial para produção de grãos, do tipo dentado e de colo- 
raçāo amarelo-alaranjada. Apresenta, ainda, variabilidade genética para tolerância a niveis tóxicos de $\mathrm{Al}^{3+}$ no solo.

A população IAC-Taitinga é constituída de $87,5 \%$ de germoplasma da populaçāo IAC-Taiúba (grãos amarelos) e 12,5\% da variedade Tuxpeño-1 (grãos brancos), sendo as plantas de porte médio, ciclo intermediário e grãos dentados brancos.

As populações IAC-lubatã (grãos amarelos) e IAC-Moroti (grãos brancos) foram derivadas de uma população de milho segregante para coloração de grãos, de base genética ampla, formada do intercruzamento de variedades e híbridos locais de grãos amarelos com variedades exóticas de grãos amarelos e brancos, resistentes ao míldio, doença fúngica causada por Peronosclerospora sorghi. Ambas têm apresentado elevada produtividade, ciclo intermediário, grãos semiduros e resistência ao mildio.

Neste estudo, foram também avaliados o híbrido interpopulacional de grãos brancos IAC-Taitinga x IAC-Moroti e seu recíproco.

A população IAC-Porangatu, de porte baixo e ciclo precoce, foi obtida por cruzamento das populações Mescla Amarillo, Amarillo Dentado DMR, Caripeño DMR, Seleción Precóz, Cupurico DMR, Tuxpeño Amarillo, Caribean DMR, Amarillo Cristalino e Guatemala DMR.

Sementes representativas das populações IAC-Taiúba, IAC-Taitinga, IAC-lubatā e IAC-Moroti e do cruzamento IAC-Taitinga x IAC-Moroti e seu reciproco foram amostradas e utilizadas para instalação dos ensaios de competição para produção e avaliaçōes tecnológicas.

Os híbridos duplos comerciais Ag-401 e C-601 e a população IAC-Porangatu foram utilizados como testemunhas nos ensaios de competição, não sendo, todavia, avaliados para os parâmetros tecnológicos devido à inexistência, na instalação do ensaio, de sementes sem tratamento fitossanitário.

No ano agrícola de 1984/85, esses materiais foram testados em ensaios de competição, quanto à produtividade e outras características agronômicas em dois locais paulistas, Mococa e Campinas. Adotou-se o delineamento de blocos ao acaso, com cinco repetiçōes, sendo cada parcela experimental constituida de duas linhas de $5 \mathrm{~m}$ de comprimento, com espaçamento de $0,90 \mathrm{~m}$ entre linhas e $0,20 \mathrm{~m}$ entre plantas dentro da linha. Assim, a área de cada parcela experimental foi de $9,00 \mathrm{~m}^{2}$, e a densidade populacional de 55.500 plantas por hectare.

Nos dois locais, efetuaram-se observações para as seguintes características agronômicas: altura da planta e de inserçāo da espiga, estande final, peso de espiga despalhada e de grãos. A análise da variância para altura da planta e da espiga foi efetuada a partir das médias dos dados obtidos para dez plantas competitivas por parcela experimental. Quanto à produção de grãos, a análise foi 
realizada após a correção do estande (ZUBER, 1942) e da umidade dos grãos, na base de $15,5 \%$.

As avaliações tecnológicas foram feitas no laboratório da Seção de Panificação do Instituto de Tecnologia de Alimentos (ITAL). Retirou-se inicialmente o gérmen, por processo manual, de uma amostra de sementes de cada população. $O$ endosperma (canjica) foi submetido ao processo de moagem, em moinho de martelo, obtendo-se o fubá mimoso, com o mesmo nivel granulométrico da farinha de trigo.

Para os testes de panificação, utilizou-se uma farinha mista (90\% de farinha de trigo e $10 \%$ de fubá mimoso), preparando-se pães do tipo forma, que foram avaliados posteriormente quanto às características físicas. Esse tipo de pão, empregado no teste, permite melhor percepção de diferenças quando se altera a composição da mistura das farinhas usadas. $\mathrm{Na}$ avaliação física dos pães, foram determinados o volume específico comparativo (V.E.C.) e o escore total comparativo (Es.T.C.). O parâmetro V.E.C. refere-se ao volume do pão feito com farinha mista em relação ao volume do pão feito somente com farinha de trigo. O Es.T.C. diz respeito a um conjunto de notas dadas às diversas características de qualidade do produto final, tais como: textura, cor do miolo e da crosta e simetria (VITT) et al., 1980).

Os dados de rendimento de fubá mimoso e gérmen, de V.E.C. e Es.T.C., expressos em porcentagem, foram transformados em arco seno $\sqrt{\% / 100}$, antes de serem submetidos à análise estatística.

\section{RESULTADOS E DISCUSSÃO}

No quadro 1 - dados médios da produtividade de grãos e altura da planta e de inserção da espiga - observam-se valores de $\mathrm{F}$ altamente significativos para todos os caracteres. Tais valores mostram o alto potencial dessas populações para produção de grãos, se comparados com aqueles obtidos para os híbridos comerciais Ag-401 e C-601.

Levando-se em conta que o híbrido C-601 e a população IAC-Porangatu são considerados de porte baixo, os demais materiais em estudo apresentaram valores médios para a altura da planta e para a inserção da espiga.

O efeito da heterose na produção de grãos dos híbridos intervarietais foi de $4,8 \%$ em relação à média dos pais. $O$ resultado foi de magnitude bem inferior aos $18,0 \%$ obtidos por LIMA (1982) e aos $12,7 \%$, por LIMA et al. (1982). Também HALLAUER \& MIRANDA FILHO (1981), analisando as heteroses observadas em 1394 cruzamentos intervarietais, chegaram a um valor de $19,5 \%$. 
QUADRO 1. Valores médios de produtividade, altura da planta e da inserçāo da espiga de populações de milho, incluindo três testemunhas. Média de cinco repetiçōes e dois locais em 1984/85

\begin{tabular}{|c|c|c|c|}
\hline Populaçōes & $\begin{array}{l}\text { Produtividade } \\
\text { de grāos }\left({ }^{1}\right)\end{array}$ & $\begin{array}{l}\text { Altura da } \\
\text { planta }\left({ }^{1}\right)\end{array}$ & $\begin{array}{l}\text { Altura da } \\
\text { espiga( }\left(^{1}\right)\end{array}$ \\
\hline & $\mathrm{kg} / \mathrm{ha}$ & $\mathrm{m}$ & $\mathrm{m}$ \\
\hline IAC-Taitinga & $10.764 \mathrm{a}$ & $2,48 a$ & $1,50 \mathrm{a}$ \\
\hline IAC-Taiúba & $9.897 a b c$ & $2,36 \mathrm{abcd}$ & $1,39 a b$ \\
\hline IAC-Moroti & $9.407 \mathrm{bc}$ & 2,35 bcd & $1,43 a b$ \\
\hline IAC-lubatā & $9.398 \mathrm{bc}$ & $2,32 d$ & $1,36 \mathrm{~b}$ \\
\hline IAC-Taitinga $\times$ IAC-Moroti & $10.625 a b$ & $2,45 \mathrm{abc}$ & $1,47 a b$ \\
\hline IAC-Moroti x IAC-Taitinga & $10.527 a b$ & $2,47 a b$ & $1,50 \mathrm{a}$ \\
\hline IAC-Porangatu & $9.024 \mathrm{c}$ & $2,19 \mathrm{e}$ & $1,23 \mathrm{c}$ \\
\hline $\mathrm{Ag}-401$ & $10.237 \mathrm{abc}$ & $2,35 \mathrm{bcd}$ & $1,51 \mathrm{a}$ \\
\hline C-601 & $9.472 b c$ & $2,13 \mathrm{e}$ & $1,12 \mathrm{c}$ \\
\hline F (locais) & n.s. & $* *$ & $\star \star$ \\
\hline$F$ (populações) & $* *$ & $* \star$ & $\star \star$ \\
\hline$F(L \times P)$ & n.s. & n.s. & n.s. \\
\hline C.V. \% & 9,44 & 3,63 & 5,93 \\
\hline
\end{tabular}

(1) Médias seguidas de letras iguais não diferem significativamente, ao nível de $5 \%$, pelo teste de Tukey.

** Significativo ao nivel de $1 \%$.

Ocorreram diferenças significativas do rendimento de fubá mimoso e de gérmen entre os materiais (Quadro 2). A população IAC-Taitinga e o híbrido intervarietal IAC-Taitinga $\times$ IAC-Moroti mostraram os valores absolutos mais altos para rendimento de fubá mimoso $(62,68$ e $62,51 \%$ respectivamente) e os menores para rendimento de gérmen $(10,45$ e 10,30\% respectivamente). $O$ conjunto de dados obtidos indica a presença de efeito maternal. Esse efeito pode ser observado através da diferença existente entre o híbrido intervarietal IAC-Taitinga x IAC-Moroti e seu reciproco. Os híbridos intervarietais apresentaram valores semelhantes aos das populações per se, que nos cruzamentos atuaram como progenitores femininos. Esse efeito pode ser observado entre a população IAC-Taitinga e o hibrido intervarietal IAC-Taitinga $\times$ IAC-Moroti e entre a população IAC-Moroti e 0 híbrido IAC-Moroti $\times$ IAC-Taitinga. Valores semelhantes para rendimento de fubá mimoso e de gérmen foram observados entre as populaçōes IAC-lubatã e IACMoroti, o que era esperado, visto terem a mesma base genética, só diferindo entre si quanto à coloração dos grãos. Embora as populações IAC-Taiúba e IAC-Taitinga tenham base genética semelhante, revelaram diferenças significativas para 
os caracteres tecnológicos estudados. Tais diferenças foram, provavelmente, determinadas pela presença de efeito maternal, originário da população Tuxpeño-1, progenitor feminino de grãos brancos, usada na formação da população IAC-Taitinga. Efeito maternal foi observado por outros autores na proporção de gérmen em relação ao peso total do grão, teor de óleo no grão e no gérmen (GARWOOD et al., 1970), bem como na concentração dos ácidos graxos (GARWOOD et al., 1970; ROCHE et al., 1971a, b).

Os dados obtidos nos testes de panificação encontram-se no quadro 3 . Os valores de $\mathrm{F}$ para o volume específico comparativo (V.E.C.) e para o escore total comparativo (Es.T.C.) mostraram diferenças significativas entre os tratamentos ao nível de $1 \%$ de probabilidade. Os valores de V.E.C. indicam que os pães produzidos com a farinha mista ( $90 \%$ de trigo $+10 \%$ de fubá mimoso) da população IAC-Taiúba foram os que mais se aproximaram $(98,0 \%)$ dos pães feitos apenas com farinha de trigo (padrão 100\%). Por outro lado, os valores de Es.T.C. revelam que os materiais de grãos brancos (IAC-Taitinga e IAC-Taitinga $\times$ IAC-Moroti) igualaram-se ao padrão. A IAC-Moroti e o híbrido IAC-Moroti x IAC-Taitinga, também de grãos brancos, apresentaram valores menores, devido a outras características da avaliação física, como a textura. Em geral, onde se utilizou milho amarelo (IAC-Taiúba e IAC-lubatã), os valores do Es.T.C. foram inferiores ao do padrão, devido à cor amarelada do miolo do pão.

QUADRO 2. Rendimentos médios de fubá mimoso e gérmen obtidos a partir de populaçōes incluindo híbridos intervarietais de milho. Média de três repetiçōes

\begin{tabular}{lcc}
\hline Populações & Fubá mimoso $\left({ }^{1}\right)$ & Gérmen $\left({ }^{1}\right)$ \\
\hline & $\%$ & $\%$ \\
IAC-Taitinga & $62,68 \mathrm{a}$ & $10,45 \mathrm{c}$ \\
IAC-Taiúba & $61,27 \mathrm{c}$ & $12,46 \mathrm{a}$ \\
IAC-Moroti & $62,02 \mathrm{abc}$ & $11,42 \mathrm{~b}$ \\
IAC-lubatã & $61,82 \mathrm{bc}$ & $11,70 \mathrm{ab}$ \\
IAC-Taitinga x IAC-Morotj & $62,51 \mathrm{ab}$ & $10,30 \mathrm{c}$ \\
IAC-Moroti x IAC-Taitinga & $61,69 \mathrm{c}$ & $11,86 \mathrm{ab}$ \\
\hline F & $* *$ & $* *$ \\
C.V.\% & 0,29 & 1,26 \\
\hline
\end{tabular}

(1) Médias seguidas de letras iguais não diferem significativamente, ao nivel de $5 \%$, pelo teste de Tukey.

** Significativo ao nivel de $1 \%$. 
QUADRO 3. Valores médios do volume específico comparativo (V.E.C.) e do escore total comparativo (Es.T.C.), de pāes preparados com $10 \%$ de fubá mimoso e $90 \%$ de farinha de trigo. Média de três repetições

\begin{tabular}{llcc}
\hline Populações & Cor do grão & V.E.C.( $\left.{ }^{1}\right)$ & Es.T.C. $\left({ }^{1}\right)$ \\
\hline & & $\%$ & $\%$ \\
IAC-Taitinga & Branco & $89,8 \mathrm{~d}$ & $100,0 \mathrm{a}$ \\
IAC-Taiúba & Amarelo & $98,0 \mathrm{a}$ & $98,5 \mathrm{~b}$ \\
IAC-Moroti & Branco & $90,9 \mathrm{~cd}$ & $98,5 \mathrm{~b}$ \\
IAC-lubatã & Amarelo & $90,9 \mathrm{~cd}$ & $98,5 \mathrm{~b}$ \\
IAC-Taitinga x IAC-Moroti & Branco & $93,3 \mathrm{bc}$ & $100,0 \mathrm{a}$ \\
IAC-Moroți x IAC-Taitinga & Branco & $94,1 \mathrm{~b}$ & $97,2 \mathrm{c}$ \\
\hline F & & $\star *$ & $* \star$ \\
C.V. \% & & 1,32 & 0,50 \\
\hline
\end{tabular}

(1) Médias seguidas de letras iguais não diferem significativamente, ao nivel de $5 \%$, pelo teste de Tukey.

** Significativo ao nivel de $1 \%$.

\section{CONCLUSÕES}

1) As populações de milho em estudo apresentaram altos potenciais para produção de grãos, se comparados com aqueles dos híbridos comerciais Ag-401 e C-601.

2) Todas as populaçōes estudadas são passiveis de utilizar com sucesso na panificação, empregando-se $90 \%$ de farinha de trigo mais $10 \%$ de fubá mimoso.

3) A população IAC-Taiúba, de grãos amarelos, apresentou o maior valor para o volume específico comparativo (V.E.C.) e somente nāo atingiu valor máximo para escore total comparativo (Es.T.C.) em vista da coloração amarelada do miolo do pão.

4) A população IAC-Taitinga e o híbrido intervarietal IAC-Taitinga $x$ IAC-Moroti, de grãos brancos, revelaram o mesmo valor para Es.T.C. que o padrāo (100\%), embora tenham mostrado V.E.C. mais baixo.

5) Observou-se efeito maternal no rendimento do fubá mimoso obtido. 


\section{SUMMARY}

\section{MAIZE POPULATIONS PERFORMANCE AS TO AGRONOMIC AND TECHNOLOGICAL CHARACTERISTICS}

White and yellow grained maize populations and some of their reciprocal crosses were evaluated as to several agronomic traits as well as to flour yield and bread characteristics. Some populations, including the hybrids under testing showed high grain yield potentials, at the same levels of commercial hybrids Ag-401 e C-601. Significant differences were recorded for plant and ear height, among the entries studied. 'IAC-Taitinga', without differing from 'IAC-Moroti' and 'IAC-Taitinga $x$ IAC-Moroti' presented the highest flour output values. 'IAC-Taiúba' showed to be superior to the other germplasms, as to the bread volume, using a composite flour (10\% corn plus $90 \%$ wheat flour). On the other hand, the population 'IAC-Taitinga' and the hybrid 'IAC-Taitinga $x$ IAC-Moroti' showed the highest scores for bread quality.

Index terms: Zea mays L., breeding program; maternal effect; baking process; mixed flour.

\section{REFERÊNCIAS BIBLIOGRÁFICAS}

GARWOOD, D.L.; WEBER, E.J.; LAMBERT, R.J. \& ALEXANDER, D.E. Effect of different cytoplasms on oil, fatty acids, plant height, and ear heigth in maize (Zea mays L.). Crop Science, Madison, 10:39-41, 1970.

HALLAUER, A.R. \& MIRANDA FILHO, J.B. Quantitative genetics in maize breeding. Ames, la., lowa States University Press, 1981. 468p.

LIMA, M. Análise de cruzamentos intervarietais de milho (Zea mays L.) no esquema cíalélico parcial. Piracicaba, ESALQ-USP, 1982. 95p. Tese. (Doutoramento)

; GIMENES-FERNANDES, N; MIRANDA FILHO, J.B.; PEREIRA, J.C.V.A. Introduction of maize (Zea mays L.) germplasms as sources for downy mildew (Peronosclerospora sorghi) resistance. Maydica, Bergamo, 27:159-168, 1982.

ROCHE, I.A.; ALEXANDER, D.E. \& WEBER, E.J. Inheritance of oleic and linoleic acids in Zea mays L. Crop Science, Madison, 11(6):856-859, 1971.

; WEBER, E.J. \& ALEXANDER, D.E. Genetic aspects of triglyceride structure in maize. Crop Science, Madison, 11(6):871-874, 1971.

VITTI, P.; LEITÃO, R.F.F.; PIZZINATTO, A. \& PENTEADO, R.L.B. Preparo de uma farinha de milho integral e desengordurada e seu uso em panificação. Boletim do ITAL, Campinas, 17(4):451-467, 1980.

ZUBER, M.S. Relative efficiency of incomplete block designs using corn uniformity trial data. Journal of the American Society Agronomy, Madison, 34:30-47, 1942. 